\title{
Communication
}

\section{Asymmetric Diffraction in Plasmonic Meta-Gratings Using an IT-Shaped Nanoslit Array}

\author{
Hee-Dong Jeong ${ }^{1}$, Seong-Won Moon ${ }^{1,2}$ and Seung-Yeol Lee ${ }^{1, *(\mathbb{D})}$ \\ 1 School of Electronic and Electrical Engineering, College of IT Engineering, Kyungpook National University, \\ Daegu 41566, Korea; heedong@knu.ac.kr (H.-D.J.); swmoon@postech.ac.kr (S.-W.M.) \\ 2 Department of Mechanical Engineering, Pohang University of Science and Technology, Pohang 37673, Korea \\ * Correspondence: seungyeol@knu.ac.kr; Tel.: +82-053-950-6608
}

Citation: Jeong, H.-D.; Moon, S.-W.; Lee, S.-Y. Asymmetric Diffraction in Plasmonic Meta-Gratings Using an IT-Shaped Nanoslit Array. Sensors 2021, 21, 4097. https://doi.org/ $10.3390 / \mathrm{s} 21124097$

Academic Editor: Daniel Ramos

Received: 3 May 2021

Accepted: 11 June 2021

Published: 14 June 2021

Publisher's Note: MDPI stays neutral with regard to jurisdictional claims in published maps and institutional affiliations.

Copyright: (c) 2021 by the authors. Licensee MDPI, Basel, Switzerland. This article is an open access article distributed under the terms and conditions of the Creative Commons Attribution (CC BY) license (https:// creativecommons.org/licenses/by/ $4.0 /)$.

\begin{abstract}
Diffraction is a fundamental phenomenon that reveals the wave nature of light. When a plane wave is transmitted or reflected from a grating or other periodic structures, diffracted light waves propagate at several angles that are specified by the period of the given structure. When the optical period is shorter than the wavelength, constructive interference of diffracted light rays from the subwavelength-scale grating forms a uniform plane wave. Many studies have shown that through the appropriate design of meta-atom geometry, metasurfaces can be used to control light properties. However, most semitransparent metasurfaces are designed to perform symmetric operation with regard to diffraction, meaning that light diffraction occurs identically for front- and back-side illumination. We propose a simple single-layer plasmonic metasurface that achieves asymmetric diffraction by optimizing the transmission phase from two types of nanoslits with Iand T-shaped structures. As the proposed structure is designed to have a different effective period for each observation side, it is either diffractive or nondiffractive depending on the direction of observation. The designed structure exhibits a diffraction angle of $54^{\circ}$, which can be further tuned by applying different period conditions. We expect the proposed asymmetric diffraction meta-grating to have great potential for the miniaturized optical diffraction control systems in the infrared band and compact optical diffraction filters for integrated optics.
\end{abstract}

Keywords: asymmetric diffraction; metasurface; nanostructured optical filter; non-reciprocal

\section{Introduction}

Because of recent growth of industrial fields related to digital holographic and compact integrated optical device technologies, the use of light diffraction as a key technology has become tremendously important. This includes light display via spatial light modulators, image sensing and the precise extraction of external information through sophisticated lens systems [1-10]. Diffraction is a representative characteristic of light waves, and it is well known that when a plane wave is transmitted or reflected in a gridded periodic structure, multiple degrees of diffraction occur at specific angles based on the diffraction law. Conversely, when the period of a system is below the diffraction limit, constructive interference of diffracted light rays from the subwavelength-scale grating forms a uniform plane wave. The light transmission and reflection characteristics are effectively determined by the geometry of the subwavelength unit structure, which is a key concept for building metasurfaces with sophisticated functions [11-15].

A metasurface is an artificially designed surface with a unit-cell geometry made up of meta-atoms, which are designed by periodically arranging the structure at the subwavelength scale to precisely control its general light-interaction properties, such as reflection/refraction [16,17], polarization [18-20], and nonlinear response [21,22]. Based on the advent of the metasurface, various issues that were difficult to overcome with geometric optics alone have been resolved, such as the development of super-resolution lenses beyond the diffraction limit and aberration-free metalenses for imaging systems [23-26]. 
Metasurfaces are generally fabricated via a well-known semiconductor fabrication process, and they can be made to be very compact, thereby assisting in the miniaturization of optical systems. Increasing numbers of studies are being reported on the application of metasurfaces to practical technologies instead of conventional optics, such as to see-through metalenses for AR and wide-viewing-angle digital holograms [27-31].

Although various types of metasurfaces for customizing the optical properties of light have been reported, most of them have symmetrical transmission, producing the same output on the front and back sides of the structure. Breaking such a symmetry to achieve what is often called the asymmetrical transmission of light may be an important technique for integrated optics, optical telecommunications, and AR displays owing to its role in optical isolation [32-34].

However, it is well known that an ideal level of optical isolation can only be achieved using a medium with nonreciprocal, nonlinear or chiral metamaterials, which require bulky optics or a complex fabrication process, respectively [35,36]. Moreover, recently, various studies have reported breaking the traditional rules of optical symmetry using metasurfaces, although they have not used nonreciprocal materials to completely violate the Lorentz reciprocity theorem [37].

For example, studies have reported asymmetric optical properties for different directions by optimizing the geometric conditions of metasurfaces, such as an asymmetricdiffraction optical device using a difference in phase delay caused by tuning the parameter of the depth of the groove [38] or an asymmetric propagation device using the difference in intensity and phase delay of electromagnetic waves caused by the lossy planar chiral structure [39]. Additionally, asymmetric transmission studies using a nanopolymerization technique, such as multichannel polarization conversion elements using anisotropic diffraction stripe structures in which nematic molecule materials are homogeneously arranged, have been reported [40].

Moreover, a study by Lawrence et al. demonstrated the non-reciprocal manipulation of near-infrared (NIR) light with ultrathin metasurfaces [41]. The proposed structure exhibited one-way transmission through a thin periodic silicon metasurface using high-quality-factor resonance dependent on the Kerr effect [42,43]. However, such an approach generally relies on ultrafast optical nonlinearities to achieve fast modulation rates. Therefore, high power is required, leading to a disadvantage in terms of power consumption.

Further, Frese et al. demonstrated a two-layer metasurface system that breaks the spatial symmetry of propagation, resulting in distinct bidirectional holographic image generation [44]. Their proposed structure has adjustable asymmetric transmission properties with full phase and amplitude modulation. This method leads to a high degree of freedom regarding tuning, but because the system is composed of two layers, it is bulky and expensive and aligning the two layers requires a high-end fabrication technique.

As previously outlined, metasurfaces with asymmetric transmission properties are being studied using various approaches. However, to the best of our knowledge, a device with a single-layer thin-film structure capable of asymmetric diffraction has not yet been reported.

In this study, we propose a novel design for a plasmonic metasurface as part of an asymmetric diffraction filter, for which the presence or absence of diffracted light can be selected according to the direction of illumination. Because the proposed structure is not designed as a nonreciprocal material or structure, it cannot completely block the output in the opposite direction. However, the proposed method enables asymmetric diffraction characteristics to be obtained without breaking the Lorentz reciprocity theorem.

In the proposed structure, the unit cell of meta-atoms is composed of closely organized I- and T-shaped slits [45-48], with the "IT-shaped" unit cell being periodically arranged. The key principle of the proposed structure is to create a difference in effective period. In particular, it is designed to be shorter than the incident wavelength for the forward illumination case, defined as that in which light illumination is through the wide part of T-shaped slits. Consequently, the plane wave is transmitted without diffraction. By contrast, 
the effective period for the backward illumination case is designed to be longer than the incident wavelength, meaning the incident light is diffracted according to the diffraction law. Because the proposed structure is able to exhibit these two optical properties with only a single layer, the optical system can be compactly designed and fabricated at a low cost. Further, the proposed diffraction filter has a diffraction angle of approximately $54^{\circ}$ for the NIR wavelength, though the diffraction angle can be selectively adjusted by changing the period between meta-atoms. In the next section, we first explain the configuration of the proposed structure. Then, we present the results regarding the simulated optical properties. Details of the optimization of the proposed device follow, after which the conclusion of our work are summarized.

\section{Basic Principle of the Proposed Structure}

A schematic of the proposed asymmetric optical diffraction structure is shown in Figure 1a. The unit cell of the proposed meta-grating structure comprises two adjacent nanoslits, one being a normal slit (I-shaped slit) and the other being a trenched slit (Tshaped slit). These are patterned on a gold film, which is deposited on a $\mathrm{SiO}_{2}$ substrate. Here, the width of the I-shaped slit is denoted by $W_{\mathrm{I}}$, and the narrower and wider areas of the T-shaped slit are denoted by $W_{\mathrm{T} 1}$ and $W_{\mathrm{T} 2}$, respectively. Similarly, the thicknesses of the narrower and wider areas of the T-shaped slit are denoted as $T_{1}$ and $T_{2}$, respectively. $P$ is the physical period of the unit cell, which indicates the intervals between I-shaped slits, with the pattern being periodically arranged.

(a)

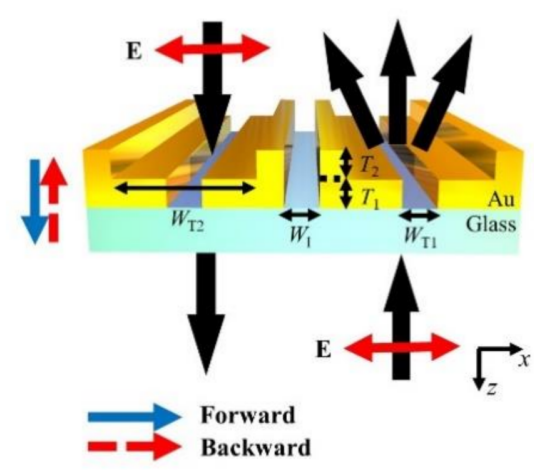

(b)

(c)
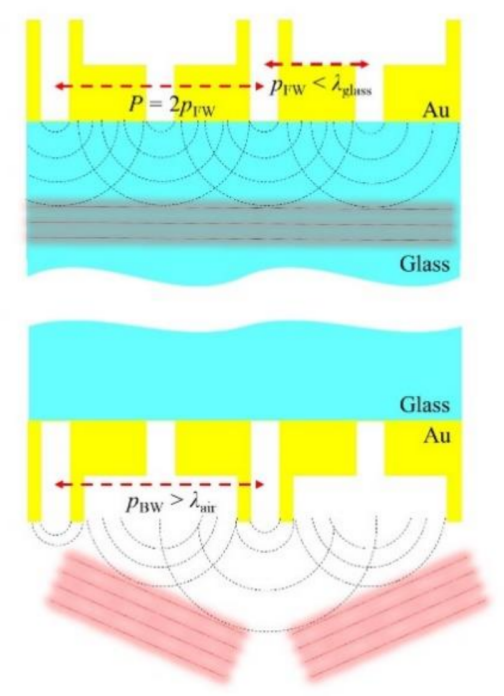

Figure 1. (a) Schematic of the proposed structure. The red dashed and blue solid lines indicate the light illumination directions with and without diffraction, respectively. $(\mathbf{b}, \mathbf{c})$ Schematic explaining the asymmetric diffraction of the proposed structure in the (b) forward and (c) backward directions.

The key concept behind obtaining asymmetric optical diffraction according to the incident light direction is to design different optical periods for forward and backward illumination ( $p_{\mathrm{FW}}$ and $p_{\mathrm{BW}}$, respectively) at the output port of the proposed structure. For the forward illumination case, $p_{\mathrm{FW}}$ is designed to be less than $\lambda_{\text {glass }}$ (Figure 1b) by optimizing the complex amplitudes of the out-coupled light from the I- and T-shaped slits to have identical values, so that the optical period of forward illumination is half of the physical period $\left(p_{\mathrm{FW}}=P / 2\right)$. Because the light transmitted through the output port of the slit generates a new point light source with an effective period shorter than the wavelength, the wavefront generated by these sources is nondiffractive. At the same time, as shown in Figure 1c, $p_{\mathrm{BW}}$ is designed to be longer than $\lambda_{\text {air }}$ because the complex amplitudes of out-coupled light are generally different from each other. Therefore, when light illumination is from the backward direction, the wavefronts generated by the I- and T- 
shaped slits are different from each other $\left(p_{\mathrm{BW}}=P\right)$, resulting in strong diffraction. Such an asymmetric diffraction depending on the illumination direction can be achieved by setting $P$ as $\lambda_{\text {air }}<P<2 \lambda_{\text {glass, }}$ and the first-order diffraction angle for backward illumination can be expressed as:

$$
\sin \theta_{\text {diff }}= \pm \frac{\lambda_{\text {air }}}{P}
$$

\section{Results and Discussion}

For our numerical analysis, we used the rigorous coupled-wave analysis (RCWA) method to calculate the full electromagnetic field distribution and diffraction efficiencies [49]. The boundaries were selected as periodic in the $x$-direction and open in the $z$-direction. The structure was assumed to be periodic and infinite in the $x$-and $y$-directions, respectively. We used NIR light with a wavelength of $980 \mathrm{~nm}$, and the light was polarized perpendicular to the slit array. For the sake of clarity, we first show the results of the proposed structure under optimized conditions. Figure $2 \mathrm{a}, \mathrm{b}$ show the simulated $H_{\mathrm{y}}$ field distribution when a Gaussian beam illuminates the structure in the forward and backward directions, respectively.
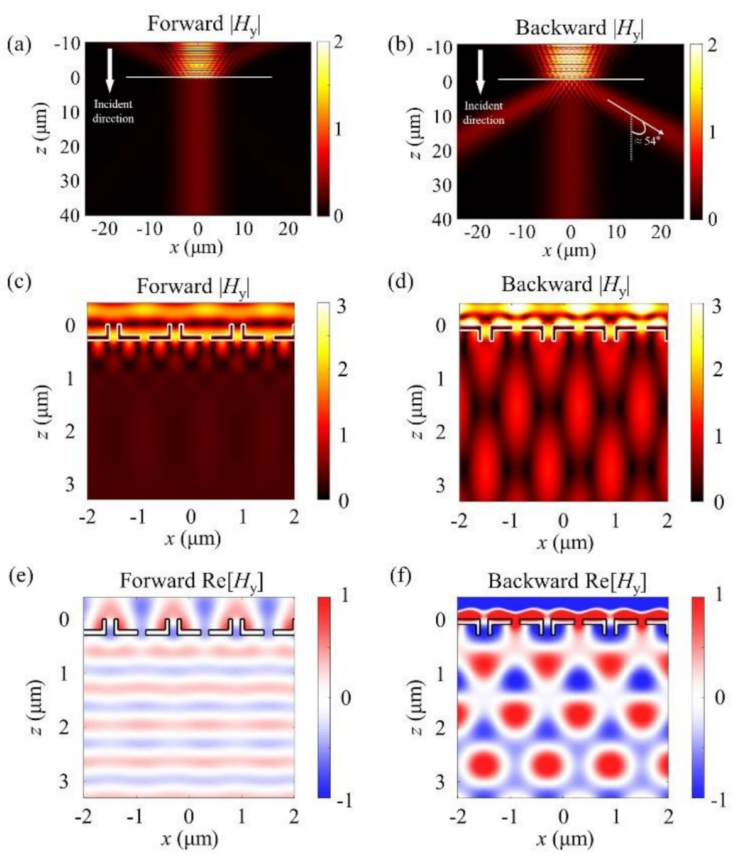

Figure 2. (a,b) $H_{\mathrm{y}}$ field distribution of (a) forward and (b) backward illumination cases when the Gaussian beam is normally incident on the proposed structure. The white arrow indicates the direction of the incident light, and the horizontal line indicates the location of the proposed structure. (c,d) Absolute value of the magnetic field in the (c) forward and (d) backward illumination cases near the nanoslit array. (e,f) Calculated real part of the magnetic field distribution (e) without diffraction (Forward $\operatorname{Re}\left[H_{\mathrm{y}}\right]$ ) and (f) with diffraction (Backward $\left.\operatorname{Re}\left[H_{\mathrm{y}}\right]\right)$.

The reason we focused on the $H_{\mathrm{y}}$ field is that the simulation conditions included no $y$-direction dependency. Therefore, only the $E_{\mathrm{x}}, H_{\mathrm{y}}$, and $E_{\mathrm{z}}$ components exist, and the two electric field components $\left(E_{\mathrm{x}}\right.$ and $\left.E_{\mathrm{z}}\right)$ could be directly calculated from the $H_{\mathrm{y}}$ field. In these simulations, the parameters $W_{\mathrm{I}}, W_{\mathrm{T} 1}, W_{\mathrm{T} 2}, T_{1}$, and $T_{2}$ were set to $150,150,900,100$, and $200 \mathrm{~nm}$, respectively. With these parametric conditions, the complex amplitudes of the Iand T-shaped nanoslits were identical for all forward directions, while those for backward directions were designed to have a phase difference of $\pi$ to maximize the diffraction efficiency. The complex amplitude can be expressed as, for example, $H_{\mathrm{y}}=\left|H_{\mathrm{y}}\right| \exp \left(\angle H_{\mathrm{y}}\right)$, where $\left|H_{\mathrm{y}}\right|$ is the absolute amplitude of the $H_{\mathrm{y}}$ field, and $\angle H_{\mathrm{y}}$ is the phase of that field. 
As shown in Figure 2a, in the forward direction, light passes through the entire surface of the proposed structure without diffraction. By contrast, Figure $2 \mathrm{~b}$ clearly shows that light transmitted through the back side is significantly diffracted, at an angle of approximately $54^{\circ}$, agreeing well with the calculation results from Equation (1).

To further investigate the field distribution of the meta-grating structure, we observed the near-field characteristics, as presented in Figure 2c-f. Figure 2c,d show the absolute amplitude of the $H_{\mathrm{y}}$ field for each illumination direction, while Figure 2e,f show the real part of the $H_{\mathrm{y}}$ field near the proposed nanoslit array. In Figure 2c,e, because the light passing through the proposed structure in the forward direction undergoes no diffraction, the absolute amplitude of the transmitted light is kept constant and the real part proceeds in the form of plane waves. It is worth noting that the out-coupled phases of the I- and T-shaped slits are almost identical, clearly indicating that the effective optical period is not the same as the physical period, instead being half of the physical period. By contrast, in the backward direction, because there is a large difference in the phase of the light passing through the two types of slits on the output side, the absolute amplitude of the transmitted light is not constant, as shown in Figure 2d. Such a phase difference is more clearly exhibited in Figure 2f, which shows the positive phase for the T-shaped slits in red and the phase for the I-shaped slits in blue.

In addition, Figure 3 shows the $E_{\mathrm{x}}$ and $E_{\mathrm{z}}$ field distribution for the forward and backward illumination cases. As shown in Figure 3a, similar to the case of the magnetic field, the constructive interference of diffracted light rays from the IT-slit array formed a uniform plane wave because it was designed to have no phase difference between the I- and T-shaped slits. Meanwhile, as shown in Figure 3b, in the backward direction, an interference pattern could be obtained from the phase difference between the I- and Tshaped slits. Moreover, Figure $3 \mathrm{c}$ depicts an image of the calculated real part of the $E_{\mathrm{z}}$ component in the forward direction. Because there was no $E_{\mathrm{z}}$ component in a plane wave propagating at an angle of $0^{\circ}$, only the near field was observed. In the backward direction (Figure 3d), the interference pattern generated by the wavefronts propagated with the 1st ordered diffraction angles.
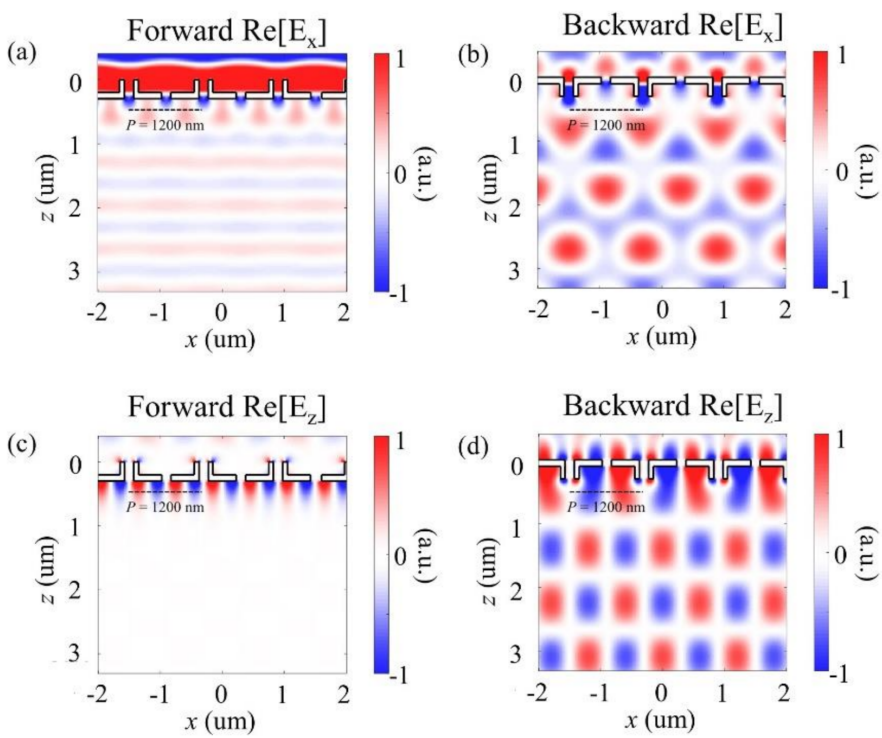

Figure 3. Real part of the $E_{\mathrm{x}}$ field distribution for (a) forward (Forward $\operatorname{Re}\left[E_{\mathrm{X}}\right]$ ) and (b) backward illumination cases (Backward $\operatorname{Re}\left[E_{\mathrm{x}}\right]$ ). The simulated real part of the $E_{\mathrm{z}}$ propagate (c) without diffraction (Forward $\operatorname{Re}\left[E_{\mathrm{Z}}\right]$ ) and (d) with diffraction (Backward $\operatorname{Re}\left[E_{\mathrm{Z}}\right]$ ) illumination on the proposed structure.

Now, we will discuss the process for determining the optimal conditions in more detail. Because a large number of parameters can be controlled, we first needed to appropriately 
classify the parameters to be controlled or fixed to efficiently achieve our goal. First, we assumed that it would be best to match the values of $W_{\mathrm{I}}$ and $W_{\mathrm{T} 1}$, as the amount of outcoupled light from the I- and T-shaped slits is generally proportional to these values, and it is necessary to match both the amplitude and phase of these slits in the forward illumination case. Second, $W_{\mathrm{I}}$ and $W_{\mathrm{T} 1}$ had to be narrow enough to allow only the fundamental metalinsulator-metal (MIM) plasmonic mode to be coupled. If higher-order MIM plasmonic modes could be coupled to these slits, the problem would become too complex to analyze. Based on these considerations, we fixed the parameters $W_{\mathrm{I}}$ and $W_{\mathrm{T} 1}$ at $150 \mathrm{~nm}$, which is narrow enough not to allow any other higher-order plasmonic modes but to be reasonably fabricated using the current focused-ion-beam milling process. We also fixed the total thickness of the overall structure $\left(T_{1}+T_{2}\right)$ at $300 \mathrm{~nm}$. Therefore, the major remaining parameters for the parameter sweep were the thickness and width of the T-shaped slits $\left(T_{2}\right.$ and $\left.W_{\mathrm{T} 2}\right)$.

To best achieve our goal of asymmetric diffraction, the first priority was the suppression of diffraction for the forward illumination case, which could only be achieved when the complex amplitudes from the I- and T-shaped nanoslits were identical for forward illumination. Figure 4a shows the difference in the out-coupled amplitude between the I- and T-shaped slits when the two parameters $\left(T_{2}\right.$ and $\left.W_{\mathrm{T} 2}\right)$ are swept. Similarly, the phase difference characteristics between the two types of slit are shown in Figure $4 \mathrm{~b}$. Based on these results, we could determine the appropriate geometric conditions for complex amplitude matching of the out-coupled light from the two types of slits for the forward illumination case, as indicated by the red "x." The plotted $H_{\mathrm{y}}$ amplitude and phase values were observed by a detector located $200 \mathrm{~nm}$ from the output slits.

(a)
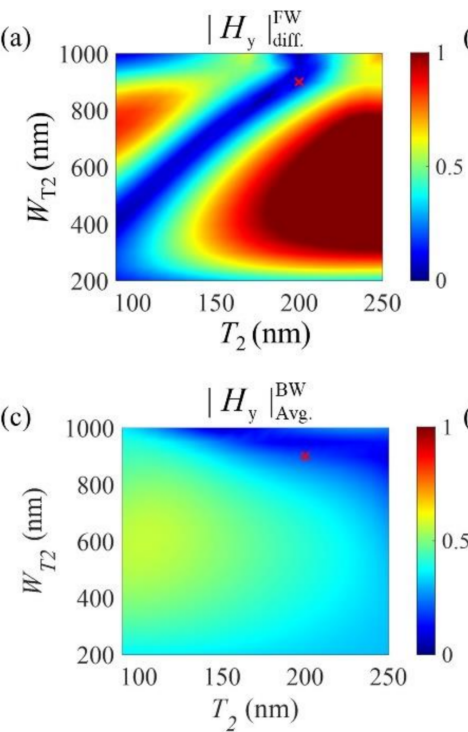
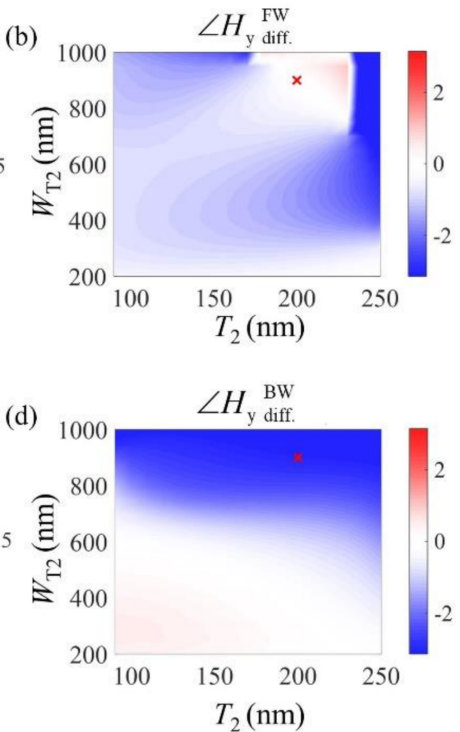

Figure 4. (a,b) Color maps of $W_{\mathrm{T} 2}$ and $T_{2}$ for (a) amplitude $\left(\left|H_{\mathrm{y}}\right|_{\mathrm{diff}}^{\mathrm{FW}}\right)$ and (b) phase differences $\left(\angle H_{\mathrm{y}} \mathrm{FW}\right.$ diff. $)$ calculated from the output of the $\mathrm{T}$ - and I-shaped slits in the forward illumination case. (c,d) Calculated (c) average of amplitude $\left(\left|H_{\mathrm{y}}\right|_{\mathrm{Avg} .}^{\mathrm{BW}}\right)$ and (d) phase difference between the two slits $\left(\angle H_{\mathrm{y}} \begin{array}{c}\mathrm{BW} \\ \text { diff. }\end{array}\right)$ in the backward illumination case. The red " $\mathrm{x}$ " indicates the conditions chosen to optimize the proposed structure.

Next, the diffraction characteristics of the backward illumination case were analyzed, including those under the optimal conditions determined for the forward direction. The parameter sweeping was performed using the same method except that the illumination was provided from the backward direction, as shown in Figure 4c,d. For the amplitude, we plotted not the difference between but rather the average value of the out-coupled amount from both slits, as the maximum diffraction efficiency would be achieved when the out-coupled lights had a high amplitude and opposite phases. As can be seen from these 
results, when the light enters in the backward direction, the phase values generated in each slit under nondiffractive conditions (red cross marked in Figure 4d) are not the same as for forward illumination but are shifted almost by a phase of $\pi$. Because the parameter sweeping results shown in Figure 4 are only strongly dependent on the variation of $T_{2}$ and $W_{\text {T2 }}$ for the forward illumination case, this asymmetric diffraction phenomenon was found to be affected by the surface plasmon polariton excited at the trench of the T-shaped slit. By contrast, because the effects of $T_{2}$ and $W_{\mathrm{T} 2}$ are quite small in the backward direction, we were able to separately design each side of the system.

In Figure 5, the sum of plus and minus first-order diffraction power efficiency $\left(D P_{+1 \text { st }}\right.$ $\left.+D P_{-1 \mathrm{st}}\right)$ under several conditions is shown to analyze the diffraction amount of the proposed structure. It is defined as the $z$-directional power coupled to the first-order diffraction versus the input power. We calculated $D P_{ \pm 1 s t}$ using Equation (2) by integrating the $z$-component of the complex Poynting vector along the single period:

$$
D P_{ \pm 1 \mathrm{st}}=\frac{\frac{1}{2} \int_{0}^{P}\left(\overrightarrow{\mathrm{E}}_{ \pm 1 \mathrm{st}} \times \overrightarrow{\mathrm{H}}_{ \pm 1 \mathrm{st}}{ }^{*}\right)_{z} d x}{\frac{1}{2} \int_{0}^{P}\left(\overrightarrow{\mathrm{E}}_{\mathrm{in}} \times \overrightarrow{\mathrm{H}}_{\mathrm{in}}{ }^{*}\right)_{z} d x}
$$

where $\overrightarrow{\mathrm{E}}_{ \pm 1 \mathrm{st}}$ and $\overrightarrow{\mathrm{H}}_{ \pm 1 \mathrm{st}}$ are the complex amplitudes of the $1^{\text {st }}$ order diffracted electromagnetic waves, and $\overrightarrow{\mathrm{E}}_{\text {in }}$ and $\overrightarrow{\mathrm{H}}_{\text {in }}$ are those of incident electromagnetic waves, respectively. Integration of the Poynting vector along a single period, 0 to $P$, will be enough to fully express the total power flow due to the periodic nature of the proposed structure. A similar definition can also be applied to define $D P_{0 \text { th }}$ by replacing 1 st order diffracted waves into 0th order diffracted waves.
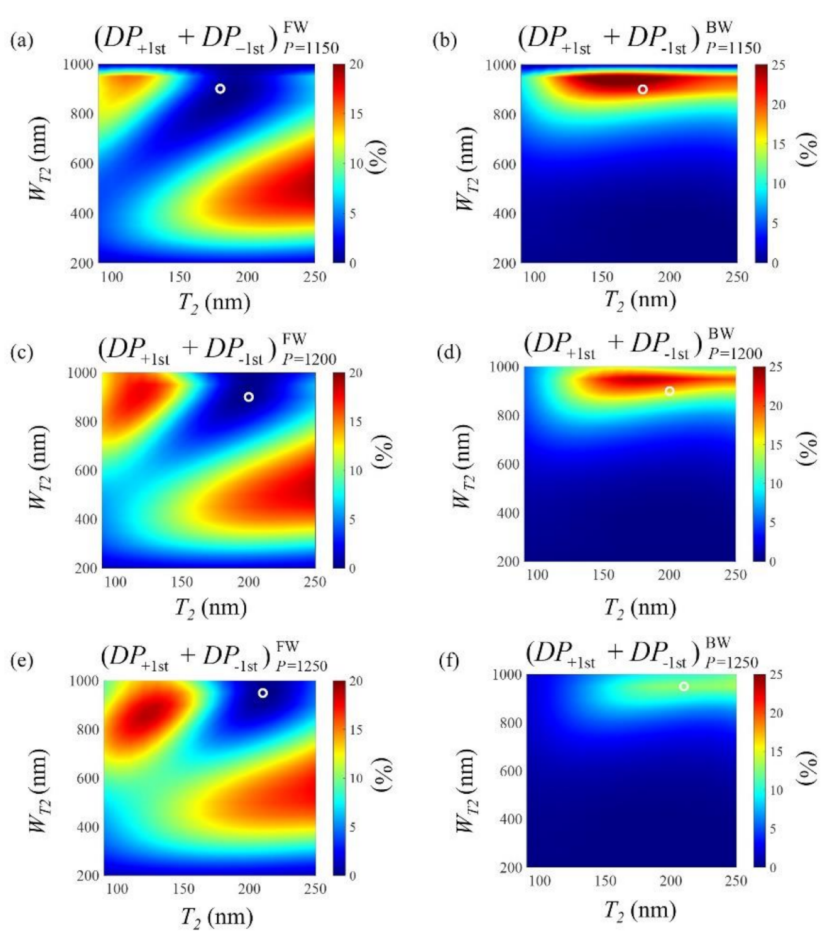

Figure 5. Various conditions for analyzing the sum of plus and minus first-order diffraction power efficiency $\left(D P_{+1 \mathrm{st}}+D P_{-1 \mathrm{st}}\right)$ according to the metal thickness designed for $(\mathbf{a}, \mathbf{b}) P=1150 \mathrm{~nm},(\mathbf{c}, \mathbf{d})$ $P=1200 \mathrm{~nm}$, and $(\mathbf{e}, \mathbf{f}) P=1250 \mathrm{~nm}$. $(\mathbf{a}, \mathbf{c}, \mathbf{e})$ show color images for the forward direction, while $(\mathbf{b}, \mathbf{d}, \mathbf{f})$ show those for the backward direction. The white circles indicate the optimal thickness at each $P$ value of the proposed structure. 
According to Equation (1), variation of the period can be used to tune the diffraction angle in backward illumination cases. However, as described in Section 2, the $\lambda_{\text {air }}<P<2 \lambda_{\text {glass }}$ condition should be satisfied to achieve asymmetric diffraction. Given our free-space wavelength of $980 \mathrm{~nm}$, the period should range from $980 \mathrm{~nm}$ to $1306 \mathrm{~nm}$. Three representative periods within this range were selected $(1150,1200$, and $1250 \mathrm{~nm}$ ), and the diffraction amount was determined for each case given variations in $W_{\mathrm{T} 2}$ and $T_{2}$.

A similar process for obtaining the optimized conditions was applied to each considered period. First, we determined the complex amplitude matching condition for the forward illumination cases and marked these with white circles. As shown in Figure 5a,c,e, each optimized condition has a near-zero $\left(D P_{+1 s t}+D P_{-1 s t}\right)$ value, regardless of the period. Meanwhile, Figure $5 \mathrm{~b}, \mathrm{~d}, \mathrm{f}$ show the $\left(D P_{+1 \mathrm{st}}+D P_{-1 \mathrm{st}}\right)$ values in the backward direction, which shows reasonably good diffraction performance compared with other nearby geometric conditions.

For the light illumination in the backward direction, several additional analyses were performed within the range of $\lambda_{\text {air }}<P<2 \lambda_{\text {glass; }}$ the corresponding results are given in Table 1, although these are not outlined in detail. As can be seen from Table 1, we were able to select appropriate design conditions according to the demands of the specific application. When only aiming for a higher transmission $\left\{D P_{0 \text { th }}+\left(D P_{+1 s t}+D P_{-1 s t}\right)\right\}$ value, the best design condition was $P=1100 \mathrm{~nm}$, although in this case, the diffraction angle was still too large. When a reasonable amount of $\left(D P_{+1 s t}+D P_{-1 \mathrm{st}}\right)$ with a strongly suppressed the $D P_{0 \text { th }}$ was desired, $P=1150 \mathrm{~nm}$ could be selected. In this way, our proposed structure can be flexibly designed to obtain the desired diffraction angle or diffraction efficiency within the allowable range. As we only studied cases in which some of our geometric parameters, such as $W_{\mathrm{I}}, W_{\mathrm{T} 1}$, and $T_{1}+T_{2}$, were considered as fixed values, the possibility of finding better conditions with additional degrees of freedom remains.

Table 1. In the case of backward illumination, detailed diffraction results within the periodic condition range satisfying the provision of asymmetric diffraction.

\begin{tabular}{cccccc}
\hline $\boldsymbol{P}[\mathbf{n m}]$ & $\boldsymbol{W}_{\mathrm{T} 2}[\mathbf{n m}]$ & $\boldsymbol{T}_{\mathbf{2}}[\mathbf{n m}]$ & $\boldsymbol{D P}_{\mathbf{0 t h}}(\mathbf{\%})$ & $\begin{array}{c}\left.\mathbf{D P}_{+\mathbf{1 s t}}+\boldsymbol{D P}_{-\mathbf{1 s t}}\right) \\
\mathbf{( \% )}\end{array}$ & $\begin{array}{c}\text { Diffraction Angle } \\
\left({ }^{\circ}\right)\end{array}$ \\
\hline 1100 & 850 & 190 & 11.88 & 15.2 & 62 \\
1150 & 900 & 180 & 0.80 & 22.16 & 58 \\
$1200^{1}$ & 900 & 200 & 7.06 & 16.86 & 54 \\
1250 & 950 & 210 & 2.42 & 12.96 & 51 \\
1300 & 900 & 220 & 0.48 & 1.84 & 49 \\
\hline
\end{tabular}

${ }^{1}$ Periodic condition of the proposed structure.

\section{Conclusions}

In summary, we have proposed a plasmonic metasurface for asymmetric diffraction using an I- and T-shaped nanoslit array. For the proposed metasurface, light with the same amplitude and phase is transmitted from the two types of slits when light illumination is in the forward direction, while the slits are designed to give the opposite phase when light is incident in the backward direction. Therefore, in the case of forward illumination, the optical period $p_{\mathrm{FW}}$ of the output slits can be considered shorter than the wavelength, whereas that in a backward direction $\left(p_{\mathrm{BW}}\right)$ is longer than the wavelength, leading to asymmetrical diffraction based on Huygens's principle. We expect the proposed structure to be widely applied to diffraction control units for optical waveguide systems, and compact optical asymmetric filters in integrated optics, and it can potentially be used as a part of $\mathrm{AR} / \mathrm{VR}$ display systems if it is optimized to operate in the visible light range.

Author Contributions: Conducted the RCWA simulation, designed the structure and writing, H.D.J.; data curation, S.-W.M.; writing, editing and supervision, S.-Y.L. All authors have read and agreed to the published version of the manuscript. 
Funding: This work was supported by the National Research Foundation of Korea (NRF) grant funded by the Korean Government Ministry of Science and ICT (No. 2017R1C1B2003585 and No. 2020R1A4A1019518), Technology Innovation Program (P20010672) funded by the Ministry of Trade, Industry \& Energy (MOTIE, Korea), and Institute of Information \& communications Technology Planning \& Evaluation (IITP) grant funded by the Korea government (MSIT) (No. 2019-0-00001, Development of Holo-TV Core Technologies for Hologram Media Services).

Institutional Review Board Statement: Not applicable.

Informed Consent Statement: Not applicable.

Conflicts of Interest: The authors declare no conflict of interest.

\section{References}

1. Chang, C.; Bang, K.; Wetzstein, G.; Lee, B.; Gao, L. Toward the next-generation VR/AR optics: A review of holographic near-eye displays from a human-centric perspective. Optica 2020, 7, 1563. [CrossRef]

2. Choi, W.-Y.; Lee, C.-J.; Kim, B.-S.; Oh, K.-J.; Hong, K.; Choo, H.-G.; Park, J.; Lee, S.-Y. Numerical Analysis on Viewing Angle Enhancement of Digital Hologram by Attaching a Pixelated-Random Phase Mask. Appl. Opt. 2020, 60, A54-A61. [CrossRef] [PubMed]

3. Chen, H.; Weng, Y.; Xu, D.; Tabiryan, N.V.; Wu, S.-T. Beam steering for virtual/augmented reality displays with a cycloidal diffractive waveplate. Opt. Express 2016, 24, 7287-7298. [CrossRef] [PubMed]

4. Wang, J.; Wang, Q.-H.; Hu, Y. Image Encryption Using Compressive Sensing and Detour Cylindrical Diffraction. IEEE Photonics J. 2018, 10, 1-14. [CrossRef]

5. Chakravarthula, P.; Dunn, D.; Aksit, K.; Fuchs, H. FocusAR: Auto-focus Augmented Reality Eyeglasses for both Real World and Virtual Imagery. IEEE Trans. Vis. Comput. Graph 2018, 24, 2906-2916. [CrossRef]

6. Kumar, M.B.; Kang, D.; Jung, J.; Park, H.; Hahn, J.; Choi, M.; Bae, J.-H.; Kim, H.; Park, J. Compact vari-focal augmented real-ity display based on ultrathin, polarization-insensitive, and adaptive liquid crystal lens. Opt. Lasers Eng. 2020, 128, 106006. [CrossRef]

7. Quan, X.; Matoba, O.; Awatsuji, Y. Single-shot incoherent digital holography using a dual-focusing lens with diffraction gratings. Opt. Lett. 2017, 42, 383-386. [CrossRef]

8. Park, J.; Lee, K.; Park, Y. Ultrathin wide-angle large-area digital 3D holographic display using a non-periodic photon sieve. Nat. Commun. 2019, 10, 1304. [CrossRef]

9. Jia, J.; Chen, J.; Yao, J.; Chu, D. A scalable diffraction-based scanning 3D colour video display as demonstrated by using tiled gratings and a vertical diffuser. Sci. Rep. 2017, 7, 44656. [CrossRef]

10. Draper, C.T.; Blanche, P.-A. Examining aberrations due to depth of field in holographic pupil replication waveguide sys-tems. Appl. Opt. 2021, 60, 1653-1659. [CrossRef]

11. Arbabi, A.; Arbabi, E.; Horie, Y.; Kamali, S.M.; Faraon, A. Planar metasurface retroreflector. Nat. Photonics 2017, 11, 415-420. [CrossRef]

12. Akram, M.R.; Ding, G.; Chen, K.; Feng, Y.; Zhu, W. Ultrathin Single Layer Metasurfaces with Ultra-Wideband Operation for Both Transmission and Reflection. Adv. Mater. 2020, 32, e1907308. [CrossRef]

13. Shirmanesh, G.K.; Sokhoyan, R.; Wu, P.C.; Atwater, H.A. Electro-optically Tunable Multifunctional Metasurfaces. ACS Nano 2020, 14, 6912-6920. [CrossRef]

14. Islam, K.M.R.; Choi, S. Compact Double-Layer FR4-based Focusing Lens Using High-Efficiency Huygens' Metasurface Unit Cells. Sensors 2020, 20, 6142. [CrossRef]

15. Hao, W.; Deng, M.; Chen, S.; Chen, L. High-Efficiency Generation of Airy Beams with Huygens' Metasurface. Phys. Rev. Appl. 2019, 11, 054012. [CrossRef]

16. Park, J.; Kang, J.-H.; Kim, S.J.; Liu, X.; Brongersma, M.L. Dynamic Reflection Phase and Polarization Control in Metasurfaces. Nano Lett. 2017, 17, 407-413. [CrossRef]

17. Chen, W.T.; Zhu, A.Y.; Sisler, J.; Huang, Y.-W.; Yousef, K.M.A.; Lee, E.; Qiu, C.-W.; Capasso, F. Broadband achromatic metasurfacerefractive optics. Nano Lett. 2018, 18, 7801-7808. [CrossRef]

18. Shibanuma, T.; Maier, S.A.; Albella, P. Polarization control of high transmission/reflection switching by all-dielectric metasurfaces. Appl. Phys. Lett. 2018, 112, 063103. [CrossRef]

19. Chen, W.T.; Zhu, A.Y.; Sanjeev, V.; Khorasaninejad, M.; Shi, Z.; Lee, E.; Capasso, F. A broadband achromatic metalens for focusing and imaging in the visible. Nat. Nanotechnol. 2018, 13, 220-226. [CrossRef]

20. Lesina, A.C.; Goodwill, D.; Bernier, E.; Ramunno, L.; Berini, P. Tunable plasmonic metasurfaces for optical phased arrays. IEEE J. Sel. Top. Quantum Electron. 2020, 27, 1. [CrossRef]

21. Schlickriede, C.; Kruk, S.S.; Wang, L.; Sain, B.; Kivshar, Y.; Zentgraf, T. Nonlinear Imaging with All-Dielectric Metasurfaces. Nano Lett. 2020, 20, 4370-4376. [CrossRef] [PubMed]

22. Lin, Z.; Huang, L.; Xu, Z.T.; Li, X.; Zentgraf, T.; Wang, Y. Four-Wave Mixing Holographic Multiplexing Based on Nonlinear Metasurfaces. Adv. Opt. Mater. 2019, 7, 1900782. [CrossRef] 
23. Lee, D.; Gwak, J.; Badloe, T.; Palomba, S.; Rho, J. Metasurfaces-based imaging and applications: From miniaturized optical components to functional imaging platforms. Nanoscale Adv. 2020, 2, 605-625. [CrossRef]

24. Xu, H.-X.; Ma, S.; Luo, W.; Cai, T.; Sun, S.; He, Q.; Zhou, L. Aberration-free and functionality-switchable meta-lenses based on tunable metasurfaces. Appl. Phys. Lett. 2016, 109, 193506. [CrossRef]

25. Aieta, F.; Genevet, P.; Kats, M.A.; Yu, N.; Blanchard, R.; Gaburro, Z.; Capasso, F. Aberration-Free Ultrathin Flat Lenses and Axicons at Telecom Wavelengths Based on Plasmonic Metasurfaces. Nano Lett. 2012, 12, 4932-4936. [CrossRef]

26. Wang, A.; Chen, Z.; Dan, Y. Planar metalenses in the mid-infrared. AIP Adv. 2019, 9, 085327. [CrossRef]

27. Lee, G.-Y.; Hong, J.-Y.; Hwang, S.; Moon, S.; Kang, H.; Jeon, S.; Kim, H.; Jeong, J.-H.; Lee, B. Metasurface eyepiece for aug-mented reality. Nat. Commun. 2018, 9, 4562. [CrossRef]

28. Li, L.; Cui, T.J.; Ji, W.; Liu, S.; Ding, J.; Wan, X.; Li, Y.B.; Jiang, M.; Qiu, C.-W.; Zhang, S. Electromagnetic reprogrammable coding-metasurface holograms. Nat. Commun. 2017, 8, 197. [CrossRef]

29. Jiang, Q.; Jin, G.; Cao, L. When metasurface meets hologram: Principle and advances. Adv. Opt. Photonics 2019, 11, 518-576. [CrossRef]

30. Lee, G.-Y.; Yoon, G.; Lee, S.-Y.; Yun, H.; Cho, J.; Lee, K.; Kim, H.; Rho, J.; Lee, B. Complete amplitude and phase control of light using broadband holographic metasurfaces. Nanoscale 2018, 10, 4237-4245. [CrossRef]

31. Zheng, G.; Mühlenbernd, H.; Kenney, M.; Li, G.; Zentgraf, T.; Zhang, S. Metasurface holograms reaching $80 \%$ efficiency. Nat. Nanotechnol. 2015, 10, 308-312. [CrossRef]

32. Xu, J.; Cheng, C.; Kang, M.; Chen, J.; Zheng, Z.; Fan, Y.-X.; Wang, H.-T. Unidirectional optical transmission in dual-metal gratings in the absence of anisotropic and nonlinear materials. Opt. Lett. 2011, 36, 1905-1907. [CrossRef]

33. Cakmakyapan, S.; Serebryannikov, A.E.; Caglayan, H.; Ozbay, E. One-way transmission through the subwavelength slit in nonsymmetric metallic gratings. Opt. Lett. 2010, 35, 2597-2599. [CrossRef]

34. Hadad, Y.; Soric, J.C.; Alu, A. Breaking temporal symmetries for emission and absorption. Proc. Natl. Acad. Sci. USA 2016, 113, 3471-3475. [CrossRef]

35. Shimizu, H.; Goto, S.; Mori, T. Optical isolation using nonreciprocal polarization rotation in Fe-InGaAlAs/InP semiconduc-tor active waveguide optical isolators. Appl. Phys. Express 2010, 3, 072201. [CrossRef]

36. Xia, K.; Nori, F.; Xiao, M. Cavity-Free Optical Isolators and Circulators Using a Chiral Cross-Kerr Nonlinearity. Phys. Rev. Lett. 2018, 121, 203602. [CrossRef]

37. Jalas, D.; Petrov, A.; Eich, M.; Freude, W.; Fan, S.; Yu, Z.; Baets, R.; Popovic, M.; Melloni, A.; Joannopoulos, J.D.; et al. What is-And what is not-An optical isolator. Nat. Photonics 2013, 7, 579-582. [CrossRef]

38. Cao, Y.; Fu, Y.; Zhou, Q.; Ou, X.; Gao, L.; Chen, H.; Xu, Y. Mechanism Behind Angularly Asymmetric Diffraction in Phase-Gradient Metasurfaces. Phys. Rev. Appl. 2019, 12, 024006. [CrossRef]

39. Fedotov, V.A.; Mladyonov, P.L.; Prosvirnin, S.L.; Rogacheva, A.V.; Chen, Y.; Zheludev, N.I. Asymmetric Propagation of Electromagnetic Waves through a Planar Chiral Structure. Phys. Rev. Lett. 2006, 97, 167401. [CrossRef]

40. Zanotto, S.; Sgrignuoli, F.; Nocentini, S.; Martella, D.; Parmeggiani, C.; Wiersma, D.S. Multichannel remote polarization control enabled by nanostructured liquid crystalline networks. Appl. Phys. Lett. 2019, 114, 201103. [CrossRef]

41. Lawrence, M.; Barton, D.R.; Dionne, J.A. Nonreciprocal Flat Optics with Silicon Metasurfaces. Nano Lett. 2018, 18, 1104-1109. [CrossRef] [PubMed]

42. Driscoll, J.B.; Astar, W.; Liu, X.; Dadap, J.I.; Green, W.M.J.; Vlasov, Y.A.; Carter, G.M.; Osgood, R.M. All-Optical Wavelength Conversion of $10 \mathrm{~Gb} / \mathrm{s}$ RZ-OOK Data in a Silicon Nanowire via Cross-Phase Modulation: Experiment and Theoretical Investigation. IEEE J. Sel. Top. Quantum Electron. 2010, 16, 1448-1459. [CrossRef]

43. Yu, Y.; Chen, Y.; Hu, H.; Xue, W.; Yvind, K.; Mork, J. Nonreciprocal transmission in a nonlinear photonic-crystal Fano structure with broken symmetry. Laser Photonics Rev. 2015, 9, 241-247. [CrossRef]

44. Frese, D.; Wei, Q.; Wang, Y.; Huang, L.; Zentgraf, T. Non-reciprocal asymmetric polarization encryption by layered plas-monic metasurfaces. Nano Lett. 2019, 19, 3976-3980. [CrossRef]

45. Ahadi, S.; Granpayeh, N. Femtosecond all-optical switching based on asymmetric plasmonic Kerr Fabry-Perot resonators. Opt. Commun. 2015, 349, 36-41. [CrossRef]

46. Zhang, X.; Li, Z.; Chen, J.; Yue, S.; Gong, Q. A dichroic surface-plasmon-polariton splitter based on an asymmetric T-shape nanoslit. Opt. Express 2013, 21, 14548-14554. [CrossRef]

47. Chen, J.; Li, Z.; Zhang, X.; Xiao, J.; Gong, Q. Submicron bidirectional all-optical plasmonic switches. Sci. Rep. 2013, 3, 1451. [CrossRef]

48. Chen, J.; Li, Z.; Yue, S.; Xiao, J.; Gong, Q. Plasmon-Induced Transparency in Asymmetric T-Shape Single Slit. Nano Lett. 2012, 12, 2494-2498. [CrossRef]

49. Hwi, K.; Junghyun, P.; Byoungho, L.; Lee, B.; Kim, H.; Park, J. Fourier Modal Method and Its Applications in Computational Nanophotonics; CRC Press: Boca Raton, FL, USA, 2017; p. 66. 